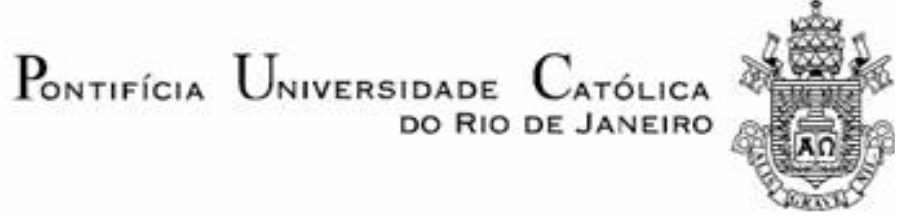

Daniel El-Jaick de Souza Mota

\title{
Análise numérica e experimental do comportamento de expansores de pele sobre base elástica
}

Dissertação apresentada como requisito parcial para obtenção do título de Mestre pelo Programa de Pós-Graduação em Engenharia Civil da PUCRio.

Orientador: Profa. Djenane Cordeiro Pamplona 


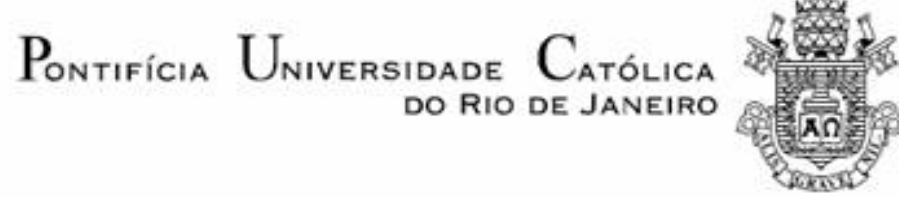

Daniel El-Jaick de Souza Mota

\section{Análise numérica e experimental do comportamento de expansores de pele sobre base elástica}

Dissertação apresentada como requisito parcial para obtenção do título de Mestre pelo Programa de PósGraduação em Engenharia Civil da PUC-Rio. Aprovada pela Comissão Examinadora abaixo assinada.

Profa. Djenane Cordeiro Pamplona

Presidente/Orientador Departamento de Engenharia Civil - PUC - Rio

Prof. Paulo Batista Gonçalves

Departamento de Engenharia Civil - PUC-Rio

Profa. Fabiana Rodrigues Leta

UFF

Dra. Stefane Rodrigues Xavier Lopes

Prof. José Eugênio Leal

Coordenador Setorial do Centro Técnico Científico - PUC-Rio

Rio de Janeiro, 13 de março de 2006 
Todos os direitos reservados. É proibida a reprodução total ou parcial do trabalho sem autorização da universidade, do autor e do orientador.

\section{Daniel El-Jaick de Souza Mota}

Graduado em Engenharia Civil pela PUC-Rio em 2003.

Ficha Catalográfica

Mota, Daniel El-Jaick de Souza

Análise numérica e experimental do comportamento de expansores de pele sobre base elástica / Daniel El-Jaick de Souza Mota ; orientador: Djenane Cordeiro Pamplona. Rio de Janeiro : PUC-Rio, Departamento de Engenharia Civil, 2006.

126 f. : il. ; $30 \mathrm{~cm}$

Dissertação (mestrado) - Pontifícia Universidade Católica do Rio de Janeiro, Departamento de Engenharia Civil.

Inclui bibliografia

1. Engenharia Civil - Teses. 2. Expansão de membranas. 3. Biomembranas. 4. Pele. 5. Base elástica. I. Pamplona, Djenane Cordeiro. II. Pontifícia Universidade Católica do Rio de Janeiro. Departamento de Engenharia Civil. III. Título. 


\section{Agradecimentos}

À minha orientadora Professora Djenane Pamplona, pela sua atenção e estímulo durante o desenvolvimento deste trabalho.

Aos professores da PUC-Rio que contribuíram para a minha formação profissional, especialmente ao Professor Raul Rosas e Silva por direcionar-me no caminho do Mestrado.

Aos funcionários do Departamento de Engenharia Civil pela maneira gentil como sempre me trataram desde a Graduação.

À turma do laboratório, Cláudio Castilho e Marcela Juliboni pela grande ajuda nas experiências realizadas.

À minha família que colaborou e me encorajou nos momentos difíceis.

Ao CNPQ e à CAPES pelo suporte financeiro. 


\section{Resumo}

Mota, Daniel El-Jaick de Souza; Pamplona, Djenane Cordeiro. Análise numérica e experimental do comportamento de expansores de pele sobre base elástica. Rio de Janeiro, 2006. 126p. Dissertação de Mestrado Departamento de Engenharia Civil, Pontifícia Universidade Católica do Rio de Janeiro.

Apesar do conhecimento do comportamento de estruturas sobre base elástica ser importante para a engenharia de forma geral, a motivação deste trabalho está focada na Biomecânica, nos nossos trabalhos envolvendo o grupo de pesquisa da Clínica do Dr.Ivo Pitanguy e da Santa Casa de Misericórdia. Em tese recente de Doutorado, foi realizada uma modelagem da técnica cirúrgica de expansão da pele. A expansão da pele é um processo fisiológico, definido como a capacidade que a pele tem de aumentar sua área superficial em resposta a uma deformação imposta. Durante as expansões acompanhadas pode-se observar que dependendo da região em que o expansor é implantado seu comportamento pode ser bastante distinto. Expansores que são implantados no couro cabeludo iniciam a expansão da pele assim que é introduzida a solução salina no expansor. Quando o expansor é implantado em regiões com mais gordura, como por exemplo na coxa, a expansão da pele não se inicia imediatamente tendo em vista que o expansor se deforma para dentro, pressionando a gordura (que oferece menos resistência) e não a pele. Este comportamento ocasiona abertura dos pontos na cicatriz e pode chegar a inviabilizar todo o processo de expansão naquela região. Para a simulação do processo de expansão da pele, foi feita a expansão com lençol de borracha e para a base elástica foi usado um conjunto que consistia em uma bexiga elástica preenchida com um material viscoso no seu interior. Este trabalho é pioneiro em investigar tanto numérica quanto experimentalmente o comportamento da expansão de lençóis de borracha sobre base rígida e base elástica, comparando $\mathrm{o}$ seu comportamento. Os resultados numéricos e experimentais da expansão do lençol de borracha tiveram uma boa concordância e a análise paramétrica da espessura do lençol de borracha comprovou a importância da obtenção mais precisa da espessura da pele pelos médicos, já que as pressões verificadas são bem diferentes para cada espessura. Foram estudadas 
diversas equações constitutivas, procurando a que melhor descrevesse o processo da expansão da pele. A formulação do material Neo-Hookeano obteve os melhores resultados para o lençol de borracha. Também foi feito um estudo com a formulação de energia de Delfino para estudar a expansão da pele humana.

\section{Palavras-chave}

Expansão de membranas; biomembranas, pele, base elástica. 


\section{Abstract}

Mota, Daniel El-Jaick de Souza; Pamplona, Djenane Cordeiro (Advisor). Numerical and experimental analysis of the behavior of skin expanders on elastic foundation. Rio de Janeiro, 2006. 126p. Msc. Dissertation Department of Civil Engineering, Pontifícia Universidade Católica do Rio de Janeiro.

Despite the knowledge of the behavior of structures on elastic base being important for the engineering of general form, the motivation of this work is focused in the Biomechanics, in our works involving the group of research of the Clinic of the Dr.Ivo Pitanguy and the Saint Casa de Misericórdia. In recent thesis of Dsc., a modeling of the surgical technique of expansion of the skin was carried through. The expansion of the skin is a physiological process, defined as the capacity that the skin has to increase its superficial area in reply to an imposed deformation. During the expansions it could be observed that depending on the region where the expander is implanted its behavior could be sufficiently distinct. Expanders who are implanted on the head initiate the expansion of the skin immediately when the saline solution is introduced in the expander. When the expander is implanted in regions with more fat, as for example in the thigh, the expansion of the skin does not initiate immediately in view of that the expander is deformed to the inner part, pressuring the fat (that offers little resistance) and not the skin. This behavior causes opening of the points in the scar and can make impracticable the process of expansion in that region. For the simulation the process of skin expansion, it was made an expansion with rubber sheet and a set was used for the elastic base that consisted of a filled elastic bladder with a viscous material in its interior. This work is pioneer in investigating numerical and experimentally the behavior of the rubber sheet expansion on rigid base and elastic base, comparing its behavior. The numerical and experimental results of the expansion of the rubber sheet had a good agreement and the parametric analysis of the thickness of the rubber sheet proved the importance of a precise measure of the skin thickness by the doctors, since the verified pressures are different for each thickness. Diverse constituent equations had been studied, looking the one that better described the process of the expansion of the skin. The 
formularization of the Neo-Hook material got the best results for the rubber sheet.

Also a study with the formularization of energy of Delfino was made to study the expansion of the human skin.

\section{Keywords}

Expansion of membranes; biomembranes, skin, elastic foundation. 


\section{Sumário}

1 Introdução 18

1.1. Objetivo da dissertação 18

1.2. Organização do texto 19

2 Revisão bibliográfica $\quad 21$

2.1. A expansão de pele 21

2.2. Expansores de pele 22

2.3. A pele humana 24

2.3.1. Composição da pele 24

2.3.2. Propriedades físicas da pele 26

2.4. Indicação da expansão de pele 26

2.5. O processo de expansão de pele 27

2.5.1. Técnicas de expansão da pele 28

2.6. Experiência com porcos 29

2.6.1. Diferentes tipos de expansores 29

2.6.2. Modificações na espessura da pele $\quad 30$

3 Formulação numérica 32

3.1. Metodologia para solução do problema 32

3.2. Formulações de energia para a borracha 32

3.2.1. Neo-Hookeano 33

3.2.2. Mooney-Rivlin 33

3.2.3. Ogden 33

3.2.4. Delfino 34

3.3. Geometria 34

3.4. Extensões principais e gradientes de deformação 36

3.5. Equações de equilíbrio da membrana 38

3.6. Base elástica 45

3.6.1. Equações de equilíbrio da membrana sobre base elástica 49 
4 Análise experimental $\quad 50$

4.1. Material utilizado 50

4.2. Preparação da membrana de borracha 52

4.3. Fixação dos elementos para a expansão 52

4.4. Ensaio para obtenção das coordenadas tridimensionais 53

4.5. Calibração do equipamento 54

4.6. Ensaio de expansão do lençol de borracha 56

4.6.1. Base rígida 56

4.6.2. Base elástica 60

4.6.2.1. Ensaio de compressão da base elástica 60

4.6.2.2. Expansão sobre base elástica 63

5 Análise numérica $\quad 68$

5.1. Método de Newton-Raphson 69

5.2. Verificação do modelo computacional 72

6 Resultados $\quad 74$

6.1. Obtenção da equação constitutiva $\quad 74$

6.1.1. Análise experimental 74

6.1.2. Análise numérica $\quad 75$

6.1.3. Comparação do modelo numérico com o modelo experimental 77

6.2. Estudo paramétrico da espessura 79

6.3. Expansão sobre base elástica 80

6.3.1. Obtenção da constante de rigidez do material que simula a base elástica $\quad 80$

6.3.2. Análise experimental $\quad 80$

6.3.3. Análise numérica 82

6.3.3.1. Expansão sobre base rígida 82

6.3.3.2. Expansão da base do expansor 83

6.3.3.3. Expansão do lençol de borracha 85

6.3.3.4. Comparação entre diferentes bases elásticas 88

6.3.4. Comparação dos resultados numéricos com os experimentais 88

6.4. Comparação entre base rígida e elástica 89 
6.5. Expansão do couro cabeludo 91

6.5.1. Expansão do couro cabeludo sobre o crânio 91

6.5.2. Expansão do couro cabeludo sobre uma camada de gordura 92

6.5.2.1. Obtenção da constante de rigidez da gordura 92

6.5.2.2. Expansão sobre a camada de gordura 92

6.5.3. Comparação da expansão sobre o crânio e sobre a camada de gordura 93

7 Conclusões 95

7.1. Sugestões 96

8 Referências Bibliográficas 97

Apêndice 1 Cálculo das coordenadas tridimensionais de uma expansão 99

A.1.1. Aparato utilizado 99

A.1.2. Realização das medições 101

A.1.2.1 Cálculo do ângulo a dos espelhos 102

A.1.2.2 Desenvolvimento dos cálculos 103

A.1.3. Validação da Metodologia 105

A.1.4. Preparação da membrana de borracha 107

A.1.5. Análise da expansão de um lençol de borracha 107

Apêndice 2 Testes de bases elásticas para a experiência 109

A.2.1 Espuma de Tradicional de Densidade D23 109

A.2.2 Espuma especial 110

A.2.3 Gelatina 111

A.2.4 Margarina dentro de bexiga 111

Apêndice 3 Relaxamento 113

Apêndice 4 Arquivos do programa Maple 9 


\section{Lista de figuras}

Figura 2.1 - Expansor redondo 23

Figura 2.2 - Diversas formas de expansores 23

Figura 2.3 - Corte longitudinal da pele $\quad 24$

Figura 2.4 - Expansão de pele 28

Figura 2.5 - Expansores redondos implantados no tórax do porco 30

Figura 3.1 - Estudo da expansão sobre base elástica 32

Figura 3.2 - Membrana indeformada 35

Figura 3.3 - Membrana deformada 36

Figura 3.4 - Tensões na membrana 38

Figura 3.5 - Modelo de Winkler 45

Figura 3.6 - Pressão dentro do expansor $\quad 48$

Figura 3.7 - Base elástica $\quad 48$

Figura 3.8 - Base elástica implementada 48

Figura 4.1 - Aparelho utilizado para medir as pressões internas 51

Figura 4.2 - Expansor redondo utilizado 51

Figura 4.3 - Anéis de acrílico onde eram fixadas as membranas de borracha 52

Figura 4.4 - Material usado para a fixação 53

Figura 4.5 - llustração do aparato 53

Figura 4.6 - Equipamento de medição para a conversão de medidas 54

Figura 4.7 - Calibração da curva de conversão do equipamento $\quad 55$

Figura 4.8 - Retirada de água do tubo de vidro 56

Figura 4.9 - Montagem do ensaio de expansão da borracha sobre base rígida 57

Figura 4.10 - Vista superior do início da expansão sobre base rígida $\quad 57$

Figura 4.11 - Vista superior do final da expansão sobre base rígida $\quad 58$

Figura 4.12 - Expansão sobre base rígida - $0 \mathrm{ml} 58$

Figura 4.13 - Expansão sobre base rígida - $50 \mathrm{ml} \quad 59$

Figura 4.14 - Expansão sobre base rígida - $100 \mathrm{ml} \quad 59$

Figura 4.15 - Expansão sobre base rígida - $150 \mathrm{ml} \quad 60$ 
Figura 4.16 - Expansão sobre base rígida - $200 \mathrm{ml} \quad 60$

Figura 4.17 - Bexiga para o ensaio de compressão 61

Figura 4.18 - Ensaio de compressão da base elástica 62

Figura 4.19 - Compressão da base elástica 62

Figura 4.20 - Bexiga com furos contendo material viscoso - Geleca $\quad 63$

Figura 4.21 - Montagem do ensaio de expansão da borracha sobre base elástica 63

Figura 4.22 - Vista superior do início da expansão sobre base elástica 64

Figura 4.23 - Vista superior do final da expansão sobre base elástica 64

Figura 4.24 - Expansão sobre base elástica - $0 \mathrm{ml}$

Figura 4.25 - Expansão sobre base elástica - $50 \mathrm{ml}$

Figura 4.26 - Expansão sobre base elástica - $100 \mathrm{ml} 66$

Figura 4.27 - Expansão sobre base elástica - $150 \mathrm{ml} \quad 66$

Figura 4.28 - Expansão sobre base elástica - $200 \mathrm{ml} \quad 67$

Figura 5.1 - Newton-Raphson $\quad 71$

Figura 5.2 - Volume x Altura $\quad 72$

Figura 5.3 - Raio x Altura - base rígida 73

Figura 6.1 - Variação do volume pela pressão intra-expansor $\quad 74$

Figura 6.2 - Média da variação do volume pela pressão intra-expansor 75

Figura 6.3 - Pnum x Volume 76

Figura 6.4 - Comparação entre diferentes formulações de energia $\quad 77$

Figura 6.5 - Comparação entre experimental e numérico 78

Figura 6.6 - Experimental x Neo-Hookeano 78

Figura 6.7 - Estudo paramétrico da espessura 79

Figura 6.8 - Ensaios de compressão $\quad 80$

Figura 6.9 - Variação do volume x Pressão interna no expansor sobre base elástica 81

Figura 6.10 - Média da variação do volume pela pressão interna no expansor sobre base elástica

Figura 6.11 - Pressão x Volume - modelo numérico da expansão do lençol de borracha sobre base rígida 82

Figura 6.12 - Geometria da expansão sobre base rígida 83

Figura 6.13 - Pressão x Volume - modelo numérico da expansão do 
lençol de borracha sobre base elástica

Figura 6.14 - Raio x Altura - da base do expansor com diferentes pressões

Figura 6.15 - Pressão x Volume - modelo numérico da expansão do lençol de borracha sobre base elástica

Figura 6.16 - Expansão - $P=0,00150623$

Figura 6.17 - Expansão - $P=0,002279699$

Figura 6.18 - Expansão - $P=0,004925779$

Figura 6.19 - Expansões sobre base elástica

Figura 6.20 - Comparação numérica entre diferentes bases elásticas 88 Figura 6.21 - Comparação da base elástica - numérico x experimental 89 Figura 6.22 - Comparação dos resultados experimentais com bases rígida e elástica

Figura 6.23 - Comparação numérica entre base rígida e elástica

Figura 6.24 - Comparação entre as curvas experimentais e numéricas das bases rígida e elástica 90

Figura 6.25 - Expansão no couro cabeludo

Figura 6.26 - Constante de rigidez da gordura

Figura 6.27 - Expansão do couro cabeludo com uma camada de gordura

Figura 6.28 - Comparação da expansão do couro cabeludo sobre o crânio e sobre uma camada de gordura

Figura A.1.1 - llustração do aparato 99

Figura A.1.2 - Foto do aparato 100

Figura A.1.3 - Aparato com semi-esfera de isopor 100

Figura A.1.4 - Esquema da reflexão no espelho 101

Figura A.1.5 - Geometria das coordenadas 102

Figura A.1.6 - Medição de 1cm sobre as "réguas de referência" (pixels) 105

Figura A.1.7 - Origens de referência (pixels) 105

Figura A.1.8 - Medição dos pontos da semi-esfera de isopor (pixels) 106

Figura A.1.9 - Medição das distâncias XB e XB' (pixels e cm) 106

Figura A.1.10 - Cálculo do z 106

Figura A.1.11 - Tabelas de comparação do cálculo do z do centro 107 
Figura A.1.12 - Gráfico z do centro (cm) versus Pressão (da Pa)

Figura A.2.1 - Carregamento do ensaio de compressão da espuma tradicional

Figura A.2.2 - Descarregamento do ensaio de compressão da espuma tradicional

Figura A.2.3 - Espuma especial

Figura A.2.4 - Carregamento do ensaio de compressão da espuma especial

Figura A.2.5 - Descarregamento do ensaio de compressão da espuma especial

Figura A.2.6 - Gelatina

Figura A.2.7 - Margarina dentro da bexiga

112

Figura A.3.1 - Gráfico pressão (da Pa) x tempo (minutos) 


\section{Lista de tabelas}

Tabela 3.1 - Modelos de Fundação

47

Tabela A.2.1 - Tabela referente ao ensaio de relaxamento 


\section{Lista de símbolos}

\section{Romanos}

$\mathrm{C}_{\mathrm{i}}$

$\mathrm{D}_{1}$

ds

$\mathrm{dS}$

$\mathrm{h}$

$\mathrm{H}$

$\mathrm{J}_{\mathrm{el}}$

$\mathrm{K}_{\mathrm{i}}$

$\mathrm{p}$

$\mathrm{P}$

$\mathrm{r}$

$\mathrm{T}_{\mathrm{i}}$

$\mathrm{x}_{\mathrm{i}}$

$\mathrm{x}_{\mathrm{i}, \mathrm{J}}$

$\mathrm{X}_{\mathrm{k}}$

Z

W

\section{Gregos}

$\phi$

$\theta$

$\sigma_{\mathrm{ii}}$

$\lambda_{\mathrm{i}}$ raio da membrana indeformada

constante elástica do material

grau de imcompressibilidade do material

comprimento deformado

comprimento indeformado

espessura da membrana deformada

espessura da membrana indeformada

deformação volumétrica elástica

curvatura principal

pressão exercida pela base elástica

pressão interna

coordenada relativa ao raio deformado

tensão principal por unidade de comprimento

coordenada de um ponto genérico no corpo deformado

gradiente de deformação

coordenada de um ponto genérico no corpo indeformado

coordenada vertical

densidade de energia de deformação

coordenada relativa ao ângulo deformado

coordenada relativa ao ângulo indeformado

coordenada relativa ao raio indeformado

tensões principais nas suas respectivas direções

extensões principais 